\title{
Solid Variant of Papillary Cystadenoma of the Epididymis Mimicking Metastatic Clear Cell Renal Carcinoma
}

\section{Abstract}

Papillary Cystadenomas of the Epididymis (PCE) are benign neoplasms of the epididymis. Their appearance can often be confused with that of a metastatic clear cell renal cell carcinoma (clear cell RCC) due to similar appearances under microscopy. Here, we report a case of a 65 year old diabetic male, who presented with a rare solid variant of PCE; whereby normal papillary or cystic structures were absent. We then performed a wider immunohistochemical profile in comparison to a similar case reported in 2014 (Yang, et al.).

Keywords: Epididymis; Neoplasms; Tumours; Haemangioma

Received: March 26, 2018; Accepted: April 03, 2018; Published: April 10, 2018

\section{Introduction}

Papillary Cystadenomas of the Epididymis (PCE) are the second most common form of benign neoplasms of the epididymis after adenomatoid tumours [1]. Despite this, the incidence of PCE is rare; with around 70 cases being reported in the literature, the first of which was by Sherrick in 1956 [2]. These tumours are often mistaken for metastatic clear cell renal carcinoma (RCC) due to the histological similarities of acinar/tubular growth, central pyknotic nuclei, clear cytoplasm, vascular stroma, and cystic or papillary architecture [3]. To further complicate matters, both tumours are associated with Von Hippel-Lindau Syndrome (VHLD). PCE usually occurs sporadically, but around a third of cases of PCE are due to VHLD, of which the majority are bilateral. Here we describe a case report for a patient with a rare variant of PCE where the normal papillary and/or cystic structures are absent and instead, the patient presents with a solid variant of the tumour.

\section{Case History}

A 65 year old obese and diabetic man presented with chronic left abdominal pain. The source of the pain was thought to be a hernia or recurrent renal stones. However, CT and IV Urograms ruled out these conditions. The patient was admitted with a sudden increase in size of a painful left scrotal swelling which he

\author{
Shaun Rees and \\ Majid Rashid* \\ Department of Pathology, Royal Gwent \\ Hospital, Newport, NP20 2UB, Wales, UK
}

\section{Corresponding author: Majid Rashid \\ Đmajid.rashid@wales.nhs.uk}

Department of Pathology, Royal Gwent Hospital, Newport, NP20 2UB, Wales, UK.

Tel: 01633234234

Citation: Rees S, Rashid M (2018) Solid Variant of Papillary Cystadenoma of the Epididymis Mimicking Metastatic Clear Cell Renal Carcinoma. J Univer Surg. Vol.6 No.2:11 described as being present for the last several months. Ultrasound showed an epididymal lesion which was removed under local anaesthesia. The clinical impression was of a haemangioma. Following surgery further imaging revealed no evidence of pelvic pathology.

\section{Radiology}

The patient was referred for an ultrasound scan. This showed both testes to be completely normal, with noted small hydroceles. On the left side within the epididymis there was a well-defined area of echogenicity with increased perfusion (Figure 1) which ruled out a cystic structure. The patient was then referred to urology to distinguish the nature of the potentially benign lesion.

\section{Pathology}

Microscopically, the epididymis was partly replaced by a welldefined clear cell tumour showing solid growth of acinar and tubular structures (Figures $\mathbf{2}$ and 3). No evidence of papillary or cystic areas could be seen. The stroma was rich in blood vessels. There was no evidence of cellular atypia or mitotic activity (Figure 4). The appearances were consistent with a solid variant of papillary cystadenoma of the epididymis (PCE), but the possibility 


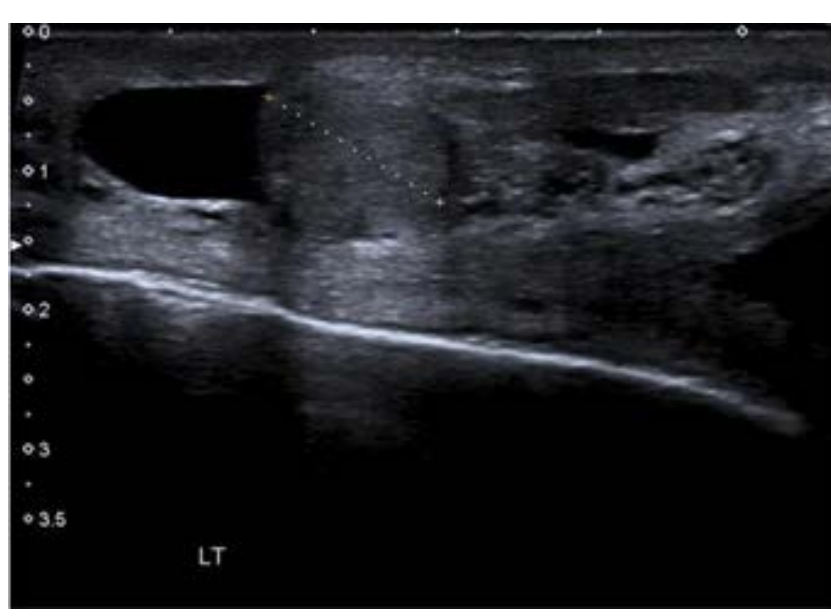

Figure 1 Ultrasound image of the Epididymal lump.

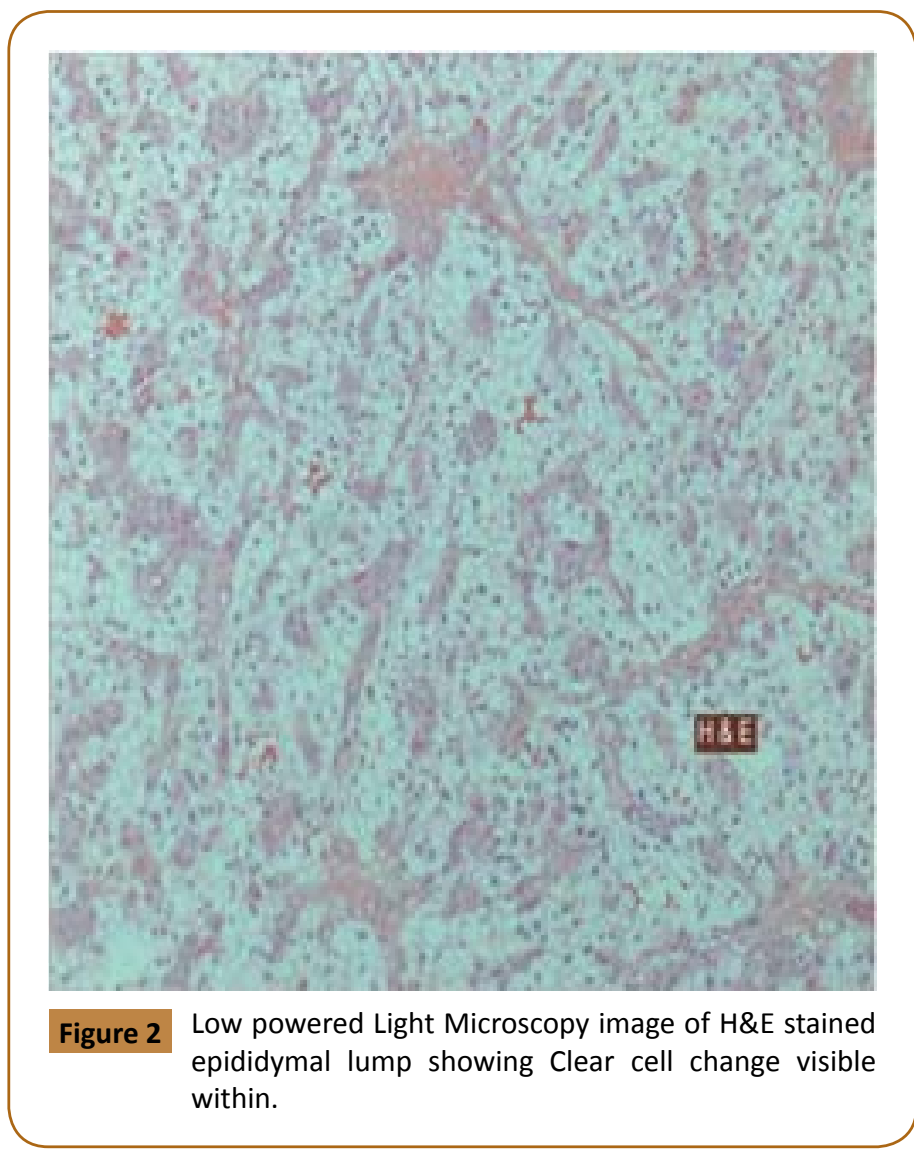

of metastatic renal clear cell carcinoma (RCC) could not be excluded. Immunohistochemically, the tumour stained positive for Cytokeratin 7 (Figure 3), Neural cell adhesion molecule (CD56), Cytokeratin AE1/AE3, Vimentin, Carbonic Anhydrase 9, and PAX8 (weak); and negative for CD10 (Figure 5) and AMACR (Racemase), Melan A, HMB45, Chromogranin, Synaptophysin, Calretinin and Inhibin. Differentiation between the two tumours was helped by CK7 positivity and CD10 and AMACR negativity for the PCE [4]. Although the diagnosis of PCE was confirmed by immunostaining, the morphological phenotype of this particular

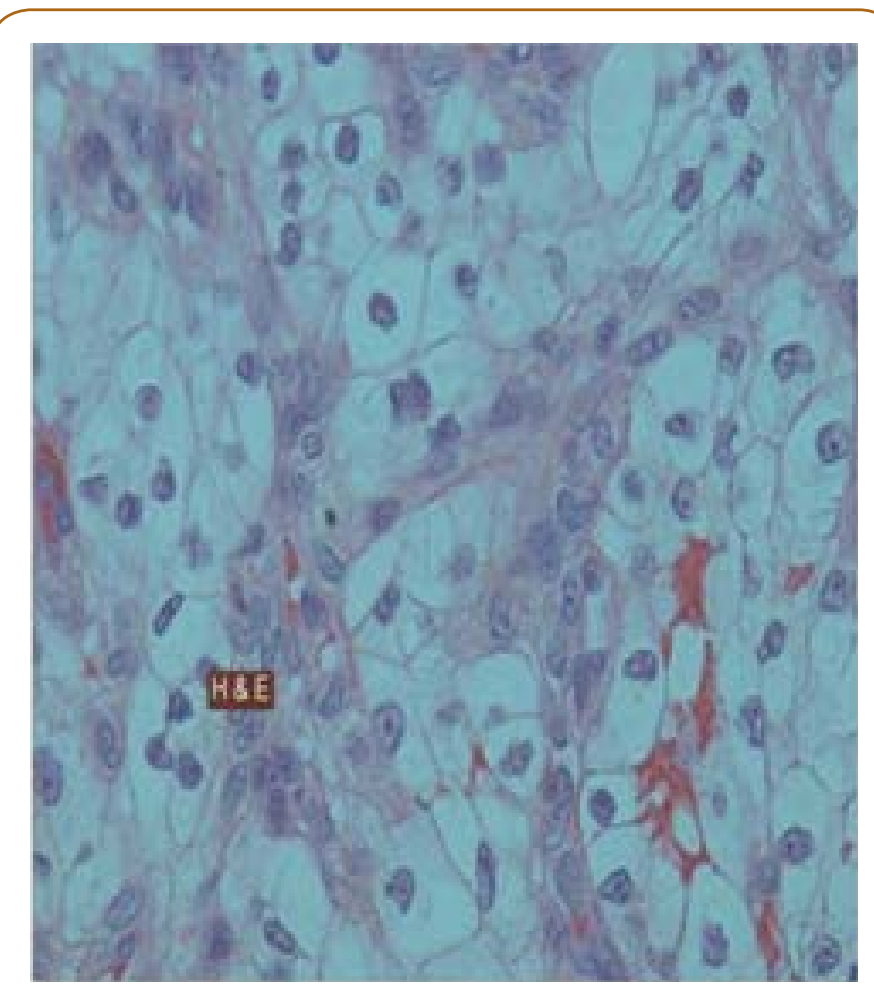

Figure 3 High powered light microscopy image of H\&E stained epididymal lump showing clear cell change visible within

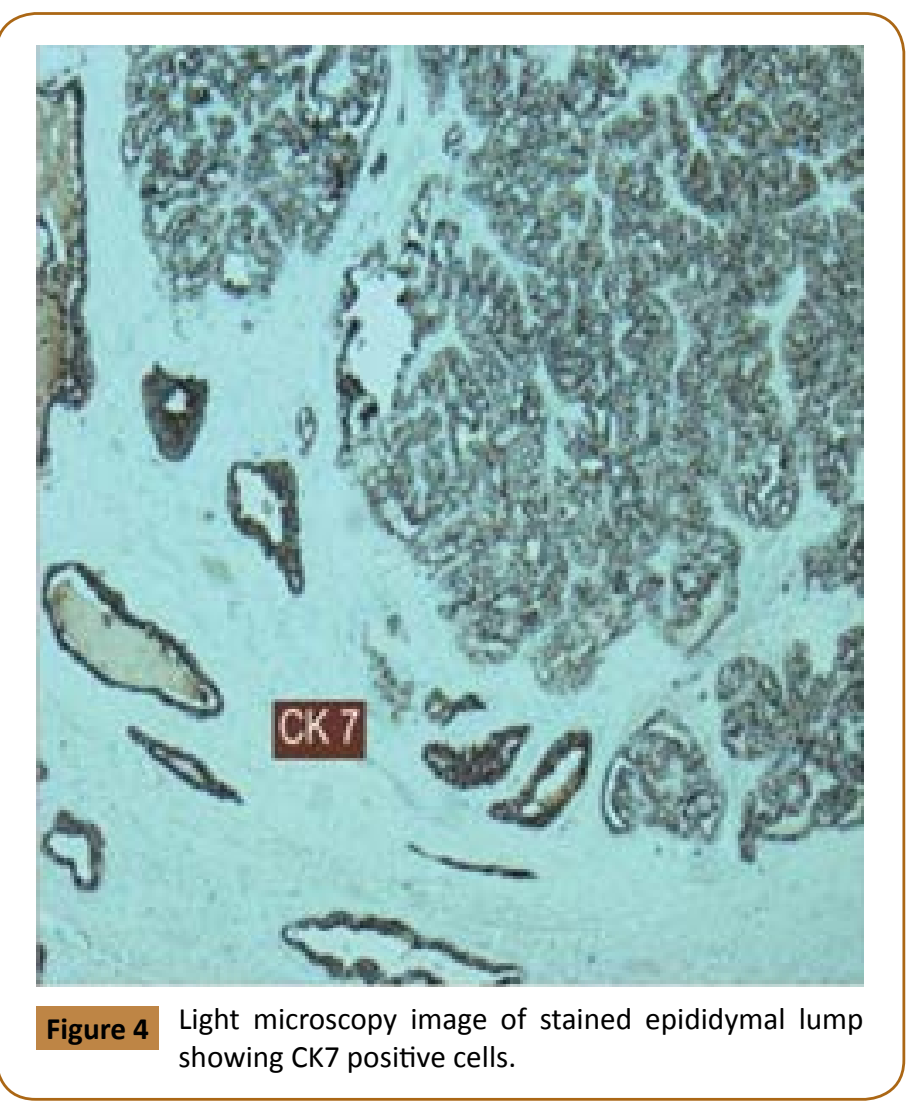

case is extremely rare. PCE is described as a papillary and cystic tumour, however, in our case these features were lacking, making it unusual. 


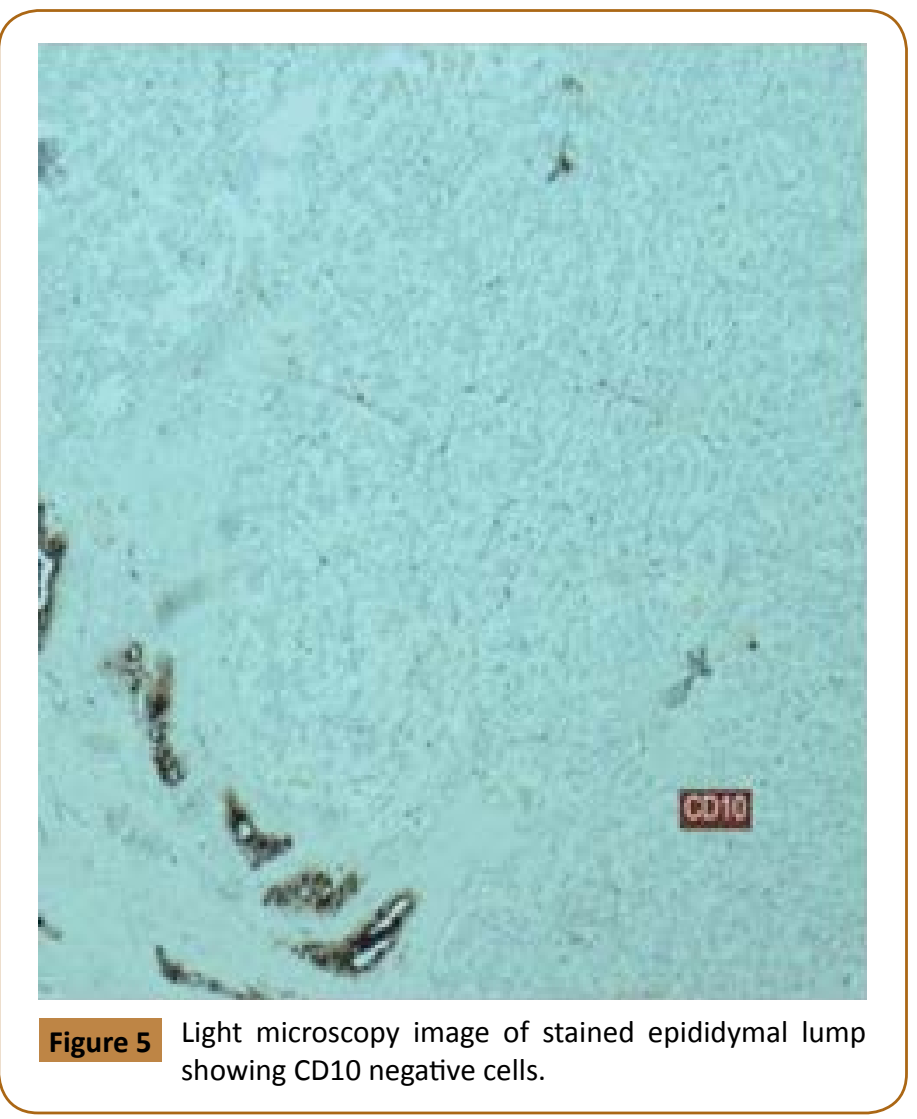

\section{Discussion}

Papillary cystadenomas (PCE) are rare epithelial tumours which are reported in the epididymis and female genital tract [4]. The majority of cases (60\%) occur sporadically and $40 \%$ are associated with von Hippel-Lindau disease (VHLD) which is an autosomal dominant tumour syndrome [3-5]. Tumours of the epididymis constitute $5 \%$ of the total intrascrotal neoplasms [6], the most common benign neoplasm is an adenomatoid tumour which is followed by rare tumours: PCE, leiomyoma, lipoma, haemangioma and melanotic neuroectodermal tumour. [1,6-8]. Malignant neoplasms are equally rare and include primary adenocarcinoma, mesothelioma and metastatic renal cell carcinoma. PCE is thought to arise from the efferent tubules of the epididymis which are of mesonephric origin $[3,6]$. Differentiation between PCE including the solid type variant and metastatic RCC (both conventional and papillary types) can be difficult. Both tumours have similar morphological features which

\section{References}

1 Toutziaris C, Kampantais S, Perdikis I, Gourvas V, Laskaridis L, et al. (2013) Papillary cystadenoma of epididymis: Is there a need for further investigation in unilateral cases?. Int J Surg Case Rep 4: 616-618.

2 Sherrick JC (1956) Papillary cystadenoma of the epididymis. Cancer 9: 403-407.

3 Odrzywolski KJ, Mukhopadhyay S (2010) Papillary cystadenoma of the epididymis. Arch Pathol Lab Med 134: 630-633. include clear cells with central pyknotic nuclei, acinar structures and vascularised stroma [5]; both originate from mesonephricmetanephric ducts; both may arise in association with VHLD with activation of angiogenesis; and both share immunoprofile with some differences [1]. This explains why PCE are often confused with metastatic RCC [6].

Unlike most described cases, our tumour was discovered in an elderly man and showed an entirely solid growth pattern with no cystic or papillary architecture. To our knowledge there is only one similar case reported in the literature of a solid variant of PCE [8]. The findings of Yang, et al. are similar to ours with cells containing bland nuclei and with a similar immunohistochemical profile (IHC) of positive staining for AE1/AE3, PAX8, CK7 and negative for CD10. However, unlike them we have performed a wider IHC profile to rule out other tumours which include: metastatic clear cell RCC (CK7 positive and AMACR and CD10 negative) [8]. This was done as Cox et al. indicated that clear cell papillary RCC show identical morphology and immunohistochemistry to PCE $[5,9]$. Stains were also used to rule out adrenal rests/adenoma (Melan A negative); adenomatoid tumour and mesothelioma (Calretinin and Inhibin negative); serous borderline tumour of para-testis which display different morphology with serous cells and papillary structures without clear cell change; and primary adenocarcinoma with marked cellular atypia and mitotic activity $[3,8]$.

The clear cell appearance of PCE is an interesting finding as the normal lining epithelium of the epididymis comprises of simple cuboidal or columnar ciliated cells which lack clear cell change. The cytoplasm of the tumour cells is filled with glycogen which stains positively with periodic acid Schiff (PAS) and negatively with diastase (PAS/D) [6]. It is well known that glycogen dissolves during processing of samples causing clearing of the cytoplasm of the tumour cells.

Due to the unilateral nature, and the fact the patient has had several renal and abdominal scans that would likely pick up cystic and/or tumour structures within the kidney, this case is considered a sporadic solid variant of PCE. These tumours are benign and the prognosis is good with rare reported cases of local recurrence after incomplete removal or malignant transformation into adenocarcinoma $[3,8]$.

\section{Acknowledgement}

A special thanks to $\mathrm{Dr}$ Richard Clements for supplying the radiology images.

4 Aydin H, Young RH, Ronnett BM, Epstein JI (2005) Clear cell papillary cystadenoma of the epididymis and mesosalpinx: Immunohistochemical differentiation from metastatic clear cell renal cell carcinoma. Am J Surg Pathol 29: 520-523.

5 Cox R, Vang R, Epstein JI (2014) Papillary cystadenoma of the epididymis and broad ligament: Morphologic and immunohistochemical overlap with clear cell papillary renal cell carcinoma. Am J Surg Pathol 38: 713-718. 
6 Yaqoob N, Ahsan A, Ahmad Z, Khan AN (2008) Clear cell papillary cystadenoma of epididymis, a mimic of metastatic renal cell carcinoma. J Pak Med Assoc 58: 209-211.

7 Kragel PJ, Pestaner J, Travis WD, Linehan WM, Filling-katz MR (1990) Papillary cystadenoma of the epididymis. A report of three cases with lectin histochemistry. Arch Pathol Lab Med 114: 672-675.
8 Yang L, Xu WS, Melamed J, Zhou M, Deng FM (2015) Solid variant of papillary cystadenoma of the epididymis. Histopathology 67: 138-141.

9 Molinié V, Balaton A, Rotman S, Mansouri D, De Pinieux I, et al. (2006) Alpha-methyl CoA racemase expression in renal cell carcinomas. Hum Pathol 37: 698-703. 